\title{
The feasibility and safety of thoracoscopic surgery under epidural and/or local anesthesia for spontaneous pneumothorax: a meta-analysis
}

\author{
Wei Chen ${ }^{1}$, Chenlei Zhang ${ }^{1}$, Gebang Wang ${ }^{1}$, Zhengjun $\mathrm{Li}^{2}$, Hailong Wang ${ }^{3}$, Hongxu Liu ${ }^{1}$ \\ ${ }^{1}$ Department of Thoracic Surgery, Cancer Hospital of China Medical University, Liaoning Cancer Hospital \& Institute, Shenyang, \\ Liaoning Province, China \\ ${ }^{2}$ Department of Thoracic Surgery, Chest Hospital of Shenyang, Shenyang, Liaoning Province, China \\ ${ }^{3}$ Department of Clinical Epidemiology and Center of Evidence Based Medicine, the First Hospital of China Medical University, \\ Shenyang, Liaoning Province, China
}

Videosurgery Miniinv 2017; 12 (3): 216-224

DOI: https://doi.org/10.5114/wiitm.2017.68895

\begin{abstract}
The aim of this study was to compare thoracoscopic surgery for spontaneous pneumothorax under epidural and/ or local anesthesia (ELA) with that under general anesthesia and prove the feasibility and safety of thoracoscopic surgery under ELA for spontaneous pneumothorax. Relevant studies were searched in five databases from their date of publication to June 2016. We collected and analyzed the data concerning operative time, hospital stay, complications, air leak, recurrence and perioperative mortality. A forest plot was performed to compare the differences between the two groups. There were no significant differences between the ELA group and the general anesthesia (GA) group in operative time, hospital stay, complications, air leak or recurrence. There were 6 deaths reported in two studies. However, patients in the ELA group had significantly shorter global operating room time. Our study demonstrated that ELA, in comparison with $G A$, is feasible and safe for thoracoscopic surgery of spontaneous pneumothorax.
\end{abstract}

Key words: thoracoscopic surgery, spontaneous pneumothorax, epidural anesthesia, local anesthesia, general anesthesia.

\section{Introduction}

Pneumothorax was first described by Itard in 1803, and then Laennec provided a further description of it in 1819 [1]. A pneumothorax is a condition in which there is excess air in the pleural cavity. Surgery is still an all-important treatment for pneumothorax, and surgical practice has also developed with the widespread adoption of video-assisted thoracoscopic surgery (VATS) rather than open thoracotomies.

In practice, the double-lumen endotracheal tube is preferred in the operation under general anesthesia (GA) to achieve single lung ventilation, in which the operative lung is deflated to make surgery convenient and the contralateral side is ventilated to supply enough oxygen for patients. However, there are various general anesthesia-related adverse effects induced by mechanical ventilation and muscle relaxants, including ventilator-induced lung injury, postoperative intractable cough, trauma to teeth, airway injuries, postoperative nausea and vomiting, an increased risk of pneumonia, impaired cardiac performance and neuromuscular problems [2-7].

\section{Address for correspondence}

Hongxu Liu, Department of Thoracic Surgery, Cancer Hospital of China Medical University, Liaoning Cancer Hospital \& Institute,

44 Xiaoheyan Road, Dadong District, Shenyang 110042, Liaoning Province, China, phone: +86 18940111218, e-mail: hongxuliu260@163.com 
Nevertheless, the appearance of epidural and/or local anesthesia (ELA) flawlessly solves these problems. In 1997, Nezu et al. first reported the role of local anesthesia with sedation in wedge resection under VATS for spontaneous pneumothorax [8]. Meanwhile, ELA was employed in more and more thoracic diseases, such as pulmonary nodule [3, 9], lung cancer [10-13], pleurodesis for malignant pleural effusion [14], lung volume reduction surgery [15], reconstruction of trachea $[16,17]$ and biopsy for anterior mediastinal masses [18].

However, there is still controversy concerning the perioperative outcomes of thoracoscopic surgery for spontaneous pneumothorax under ELA versus under GA. Nezu et al. reported that compared with GA, ELA offered the benefit of shorter operative time [8], but it was not confirmed by Pompeo et al. [19]. Nezu et al. reported that ELA has a shorter postoperative hospital stay [8], but Noda et al. held the opposite opinion [20]. We performed a systematic review and meta-analysis to comprehensively estimate the feasibility and safety of thoracoscopic surgery under epidural and/or local anesthesia for spontaneous pneumothorax.

\section{Material and methods}

\section{Strategy of literature search}

A series of relevant publications were searched using PubMed, Cochrane Library, China National Knowledge Infrastructure (CNKI), CQVIP, Wanfang databases and Google Scholar, from their date of publication to June 2016. For the integrality of the search strategy, we used "video-assisted thoracoscopic surgery", "VATS”, "general anesthesia”, "epidural anesthesia", "local anesthesia", "intubated anesthesia", "nonintubated anesthesia", "awake anesthesia", "pneumothorax" and "spontaneous pneumothorax" as key words and MeSH terms. The relevant references of all retrieved articles were further searched to determine if they were eligible.

\section{Inclusion and exclusion criteria}

Available studies were included by the following criteria: 1. randomized controlled trial (RCT) or case-control studies; 2. thoracoscopic surgery for pneumothorax; 3. comparing surgery under epidural and/or local anesthesia (ELA group) with surgery under general anesthesia (GA group); 4. except an- esthesia, the same surgical procedures were performed in all patients of each study; 5 . meaningful data about operative time, global operating room time, hospital stay, complications, air leak, recurrence or perioperative mortality could be acquired, 6. published in English or Chinese.

The following exclusion criteria were applied: 1. case reports, reviews, meta-analysis, abstracts or conference reports; 2. not comparing ELA group with GA group; 3 . the treatments of ELA group and GA group were different; 4 . lack of the relevant data we needed.

\section{Data extraction and quality assessment}

From each study, we carefully collected the useful information: first author, publication year, study period, study design, number of patients, age and gender of the patients. The data including operative time, global operating room time, hospital stay, complications, air leak, recurrence and perioperative mortality were extracted to be analyzed in this study. The randomized controlled trial was assessed by using the Jadad scale [19]. The Newcastle-Ottawa scale (NOS) was used for quality assessment of the observational studies [8, 20-22].

\section{Statistical analysis}

The differences of studies were estimated by forest plot. With a view to the relatively large difference of the means of outcomes, the standardized mean difference (SMD) was used to analyze the continuous variables [23]. Odds ratios (OR) were used to assess the values of dichotomous variables. The heterogeneity of the results of the included studies was estimated by $\chi^{2}$ and $R^{2}$. If $p>0.10$, these studies were deemed to exhibit homogeneity and a fixed effect analysis model was used. When $p<0.10$ and $R^{2}<50 \%$, these studies were considered to exhibit heterogeneity but the heterogeneity could be accepted, and a fixed effect analysis model was used too. When $p<0.10$ and $R^{2}>50 \%$, the heterogeneity was too high to be accepted, and a random effect analysis model was used. All $p$-values were two-sided and $p<0.05$ was considered significant. Publication bias was estimated by the funnel plot, and Begg's test and Egger's test were used to measure asymmetry in funnel plots [24]. All statistical analysis was performed using the STATA 12.0 package (Stata Corp, College Station, TX, USA). 


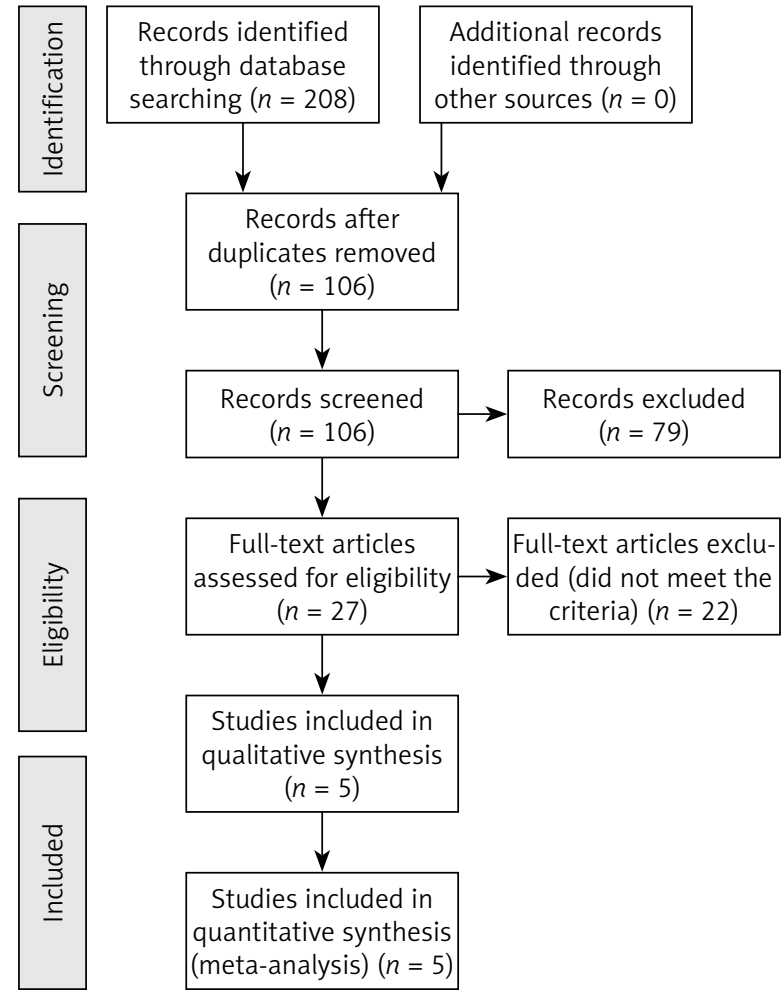

Figure 1. The flow diagram of the procedure of selecting relevant studies

\section{Results}

\section{Characteristics of studies}

The process of article search is shown in Figure 1. In all, from five electronic databases, plus Google Scholar, 208 studies were extracted. Among them, five databases provided all, but there were no additional related studies. After removing repetition and irrelevant studies by carefully screening their abstract or full texts, five studies, with a total of 419 patients, met all criteria and were included in our final analysis. Table I shows a summary of them, and the period of the publication year of these studies was from 1997 to 2016. Four studies observational studies, and one study was a randomized controlled trial. The main characteristics and perioperative information of all studies included in our analysis are presented in Tables II and III, respectively.

\section{Operative time and global operating room time}

The data of operative time were reported in all five studies [8, 19-22]. The heterogeneity among these studies was significant $\left(p=0.002\right.$ and $R^{2}=$ $76.9 \%$ ) and a random effect analysis model was used. There was no difference between the ELA group and GA group (SMD $=-0.29 ; 95 \% \mathrm{Cl}:-0.79$ to $0.20 ; p=0.246$ ) (Figure 2). Only two studies [19, 20] included the result of global operating room time. Because of the homogeneity of the two studies $\left(p=0.718\right.$ and $\left.l^{2}=0 \%\right)$, a fixed effect analysis model was adopted. We found that compared with the GA group, the ELA group had a shorter global operating room time and there was a significant difference between the two groups (SMD = -1.43; 95\% Cl: -1.90 to $-0.96 ; p<0.0001$ ) (Figure 3).

\section{Hospital stay}

The data of hospital stay were reported in all five studies [8, 19-22]. Heterogeneity was observed $\left(p<0.0001\right.$ and $\left.l^{2}=81.3 \%\right)$, so a random effect analysis model was selected. No difference was found between the ELA group and GA group (SMD $=-0.41$; $95 \% \mathrm{Cl}:-0.97$ to $0.14 ; p=0.146$ ) (Figure 4).

\section{Complications and air leak}

The data of complications were reported in all five studies [8, 19-22]. A fixed effect analysis model was used on account of the lack of significant heterogeneity $\left(p=0.841\right.$ and $\left.l^{2}=0 \%\right)$, and there was no dif-

Table I. Summary of all studies included in our analysis

\begin{tabular}{|lcccc|}
\hline Author & Publication year & Study period & Study design & Quality assessment \\
\hline Nezu et al. & 1997 & $1992-N R$ & Observational study & NOS: 7 stars \\
\hline Pompeo et al. & 2007 & $2001-2005$ & Randomized controlled trial & Jadad score: 3 points \\
\hline Noda et al. & 2012 & $2005-2010$ & Observational study & NOS:7 stars \\
\hline Ahn et al. & 2016 & $2006-2014$ & Observational study & NOS:7 stars \\
\hline Guo et al. & 2016 & $2011-2015$ & NOS:7 stars & \\
\hline
\end{tabular}

NOS - Newcastle-Ottawa scale, NR - not reported. 

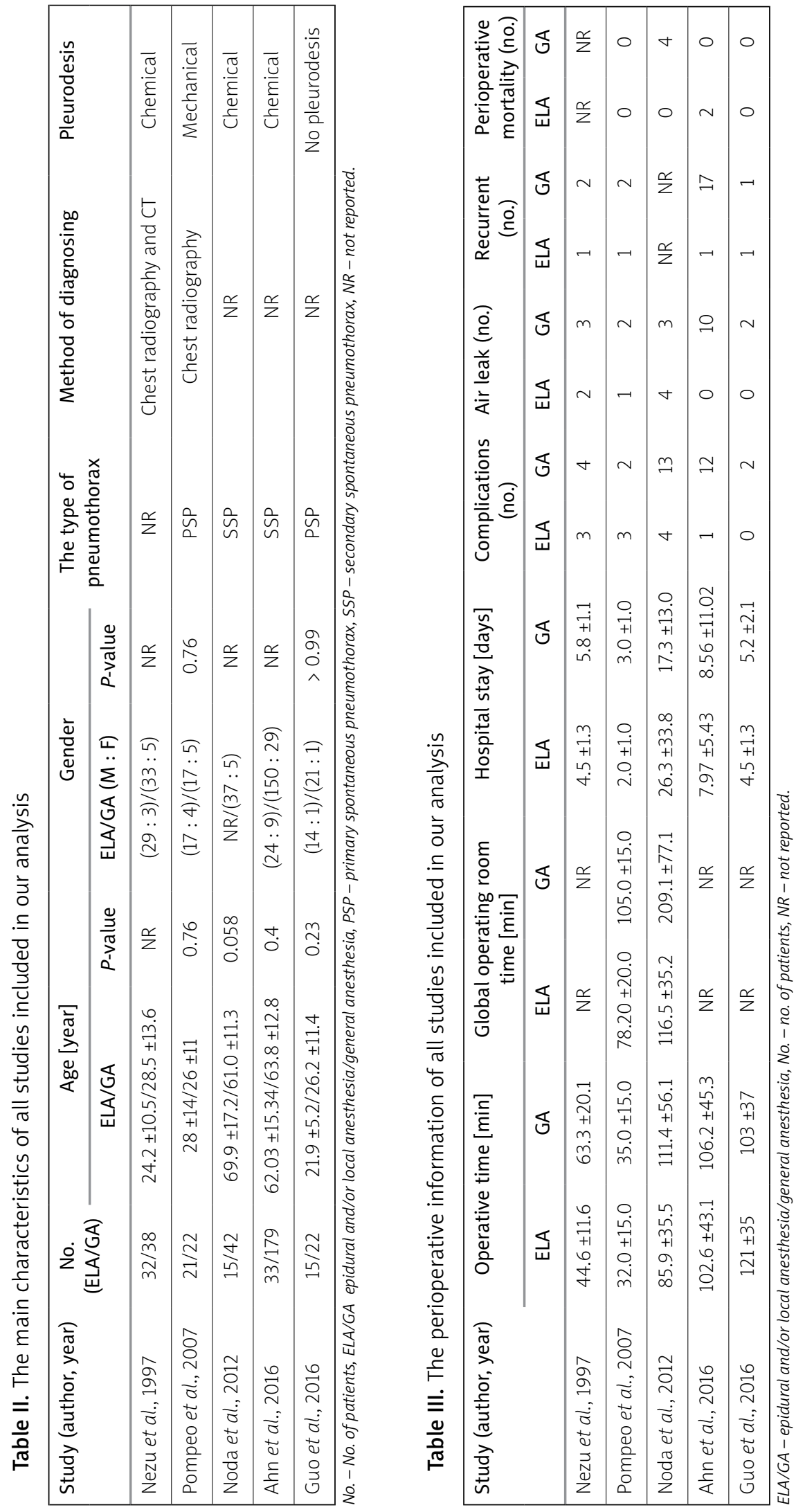


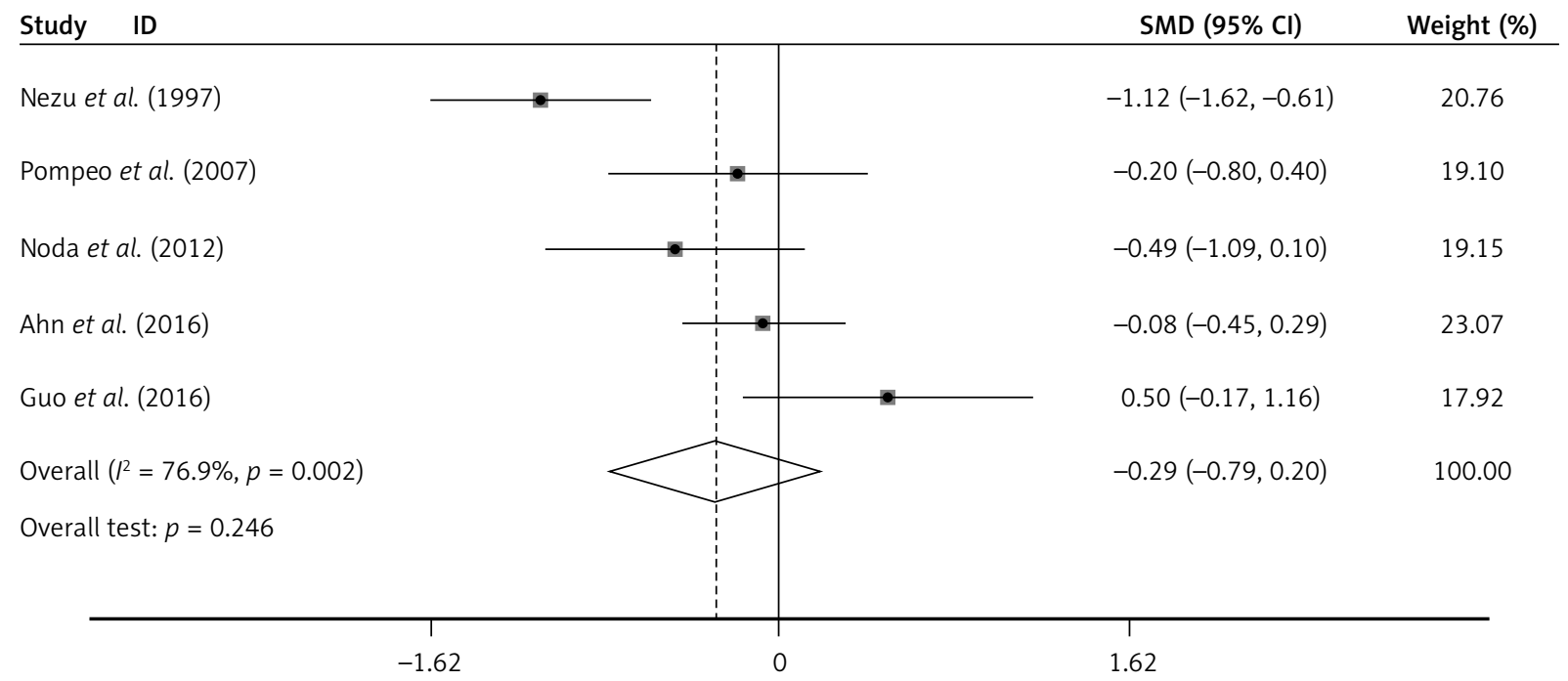

Figure 2. The forest plot of SMD and its $95 \% \mathrm{Cl}$ of operative time between ELA group ELA group and GA group Note: weights are from random effects analysis.

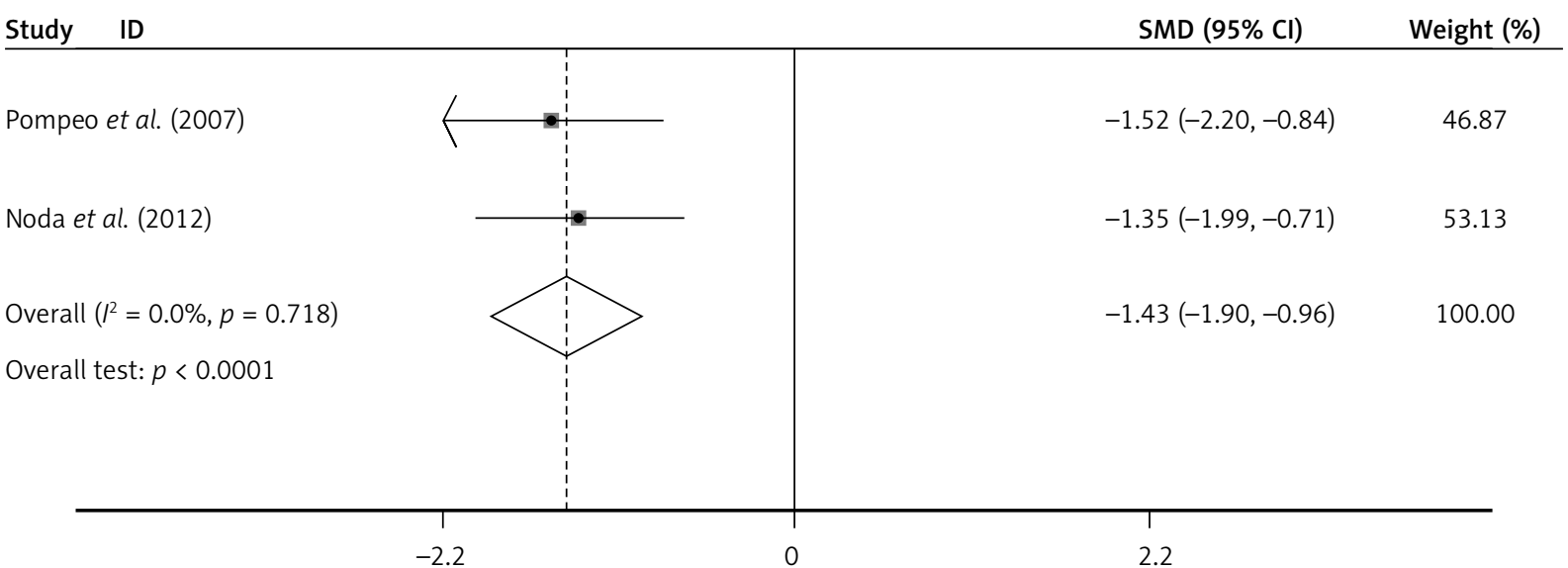

Figure 3. The forest plot of SMD and its $95 \% \mathrm{Cl}$ of global operating room time between ELA group and GS group

ference between the groups ( $\mathrm{OR}=0.76 ; 95 \% \mathrm{Cl}: 0.35$ to $1.63 ; p=0.483$ ) (Figure 5). All the included studies reported the information of air leak that is one of the complications after the surgery of pneumothorax $[8,19-22]$. Due to the homogeneity among these studies $\left(p=0.230\right.$ and $\left.R^{2}=28.7 \%\right)$, a fixed effect analysis model was employed. No statistically significant difference was found $(\mathrm{OR}=0.89 ; 95 \% \mathrm{Cl}: 0.37$ to $2.13 ; p=0.797$ ) (Figure 6).

\section{Recurrence and perioperative mortality}

The data of recurrence were reported in four studies $[8,19,21,22]$. Because of the homogeneity among these studies ( $p=0.840$ and $l^{2}=0 \%$ ), a fixed effect analysis model was used and sug- gested no difference between the ELA group and $\mathrm{GA}$ group $(\mathrm{OR}=0.49 ; 95 \% \mathrm{Cl}: 0.15$ to $1.55 ; p=$ 0.223) (Figure 7). The four studies [19-22] reported the related information of perioperative mortality and 6 deaths were recorded in two studies $[20,21]$. Of them, 2 came from the ELA group [21] and 4 from the GA group [20]. Concerning their cause of death, Yeong Ahn et al. [21] reported that one died of pneumonia and the other one was underlying lung disease, and Noda et al. [20] reported pneumonia in 2 patients and ARDS in 2 patients.

\section{Discussion}

With the progress of surgical techniques and equipment, VATS, compared with thoracotomy, un- 


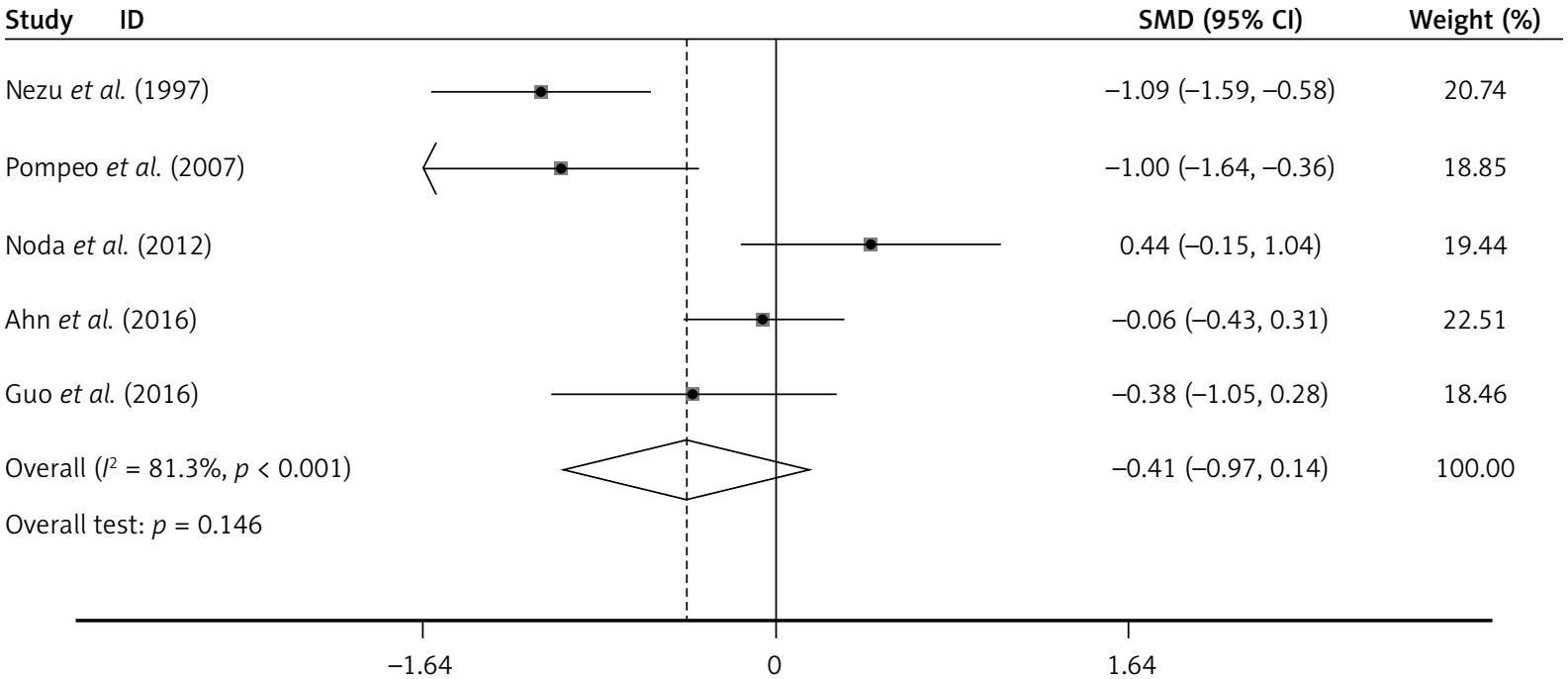

Figure 4. The forest plot of SMD and its $95 \% \mathrm{Cl}$ of hospital stay between ELA group and GA group Note: weights are from random effects analysis.

\begin{tabular}{|c|c|c|}
\hline Study ID & OR $(95 \% \mathrm{Cl})$ & Weight (\%) \\
\hline Nezu et al. (1997) & $0.88(0.18,4.26)$ & 21.22 \\
\hline Pompeo et al. (2007) & $1.67(0.25,11.13)$ & 10.72 \\
\hline Noda et al. (2012) & $0.81(0.22,3.03)$ & 32.13 \\
\hline Ahn et al. (2016) & $0.43(0.05,3.46)$ & 23.20 \\
\hline Guo et al. (2016) & $0.26(0.01,5.91)$ & 12.73 \\
\hline Overall $\left(P^{2}=0.0 \%, p=0.841\right)$ & $0.76(0.35,1.63)$ & 100.00 \\
\hline Overall test: $p=0.483$ & & \\
\hline$T$ & $T$ & \\
\hline 0.0118 & 84.5 & \\
\hline
\end{tabular}

Figure 5. The forest plot of $\mathrm{OR}$ and its $95 \% \mathrm{Cl}$ of complication between ELA group and GA group

doubtedly provides patients with minimally invasive incision and is becoming the main surgical method in thoracic surgery. With VATS under epidural and/ or local anesthesia, even less damage was provided for patients. It has become more and more popular.

Recently, a variety of studies have focused on the role of ELA in thoracic surgery. In previous studies, ELA has been shown to be suitable for various chest diseases, including pneumothorax [8, 19-22], lung cancer [10-13], pleurodesis for malignant pleural effusion [14], lung volume reduction surgery [15] and so on. Secondly, it has been proved that VATS under epidural and/or local anesthesia could be performed safely for some high-risk patients [25, 26]. Furthermore, ELA avoids or reduces the incidence of ventilator-induced lung injury, trauma to teeth, airway injuries, pneumonia, impaired cardiac performance, etc [2-7]. Water and food intake after the operation can be resumed within a few hours in the ELA group [27]. And better or similar pain scores could be gained $[3,19,28]$.

However, ELA still correlates with some potential complications, including unsatisfactory exposure of the surgical field, the emotional stress of patients due to awake surgery, dyspnea even with using the air-locking trocar, coughing reflexes during lung manipulation, hyperventilation induced by permissive 


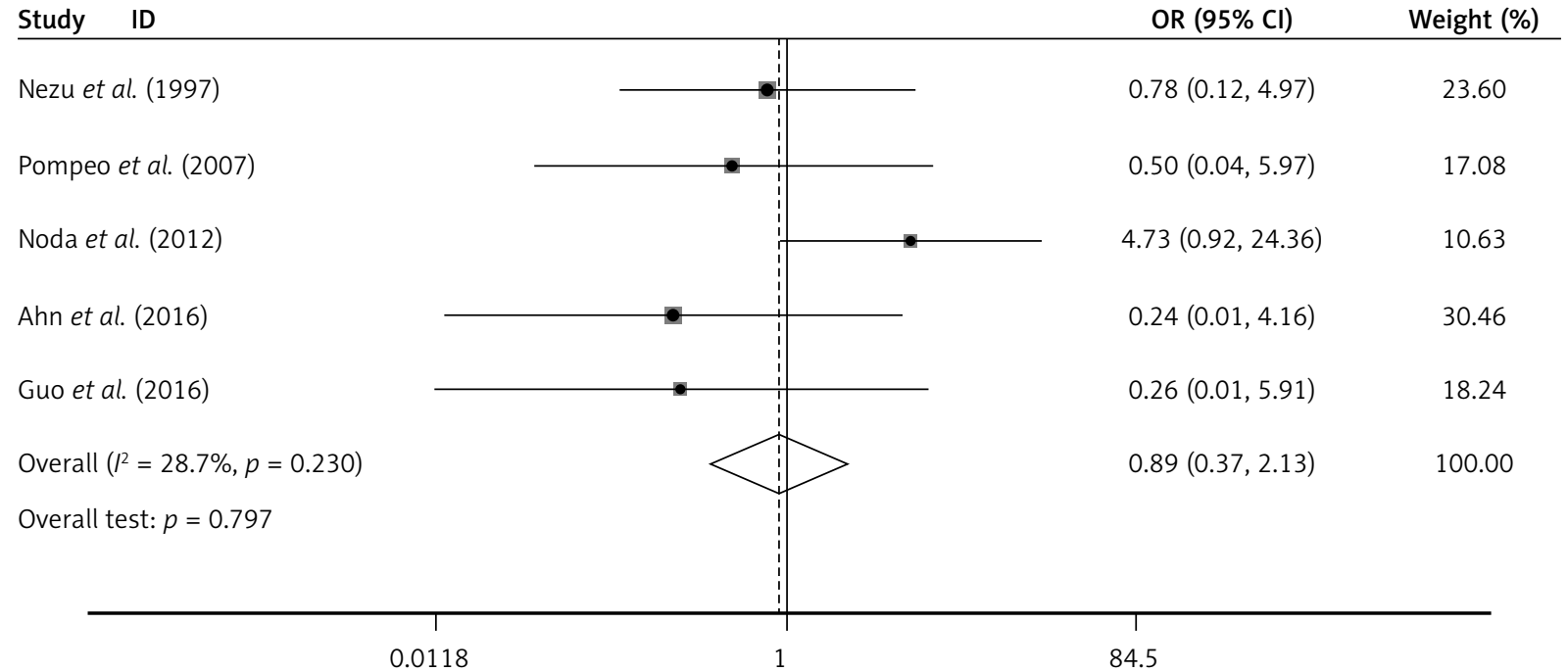

Figure 6. The forest plot of OR and its $95 \% \mathrm{Cl}$ of air leak between ELA group and GA group

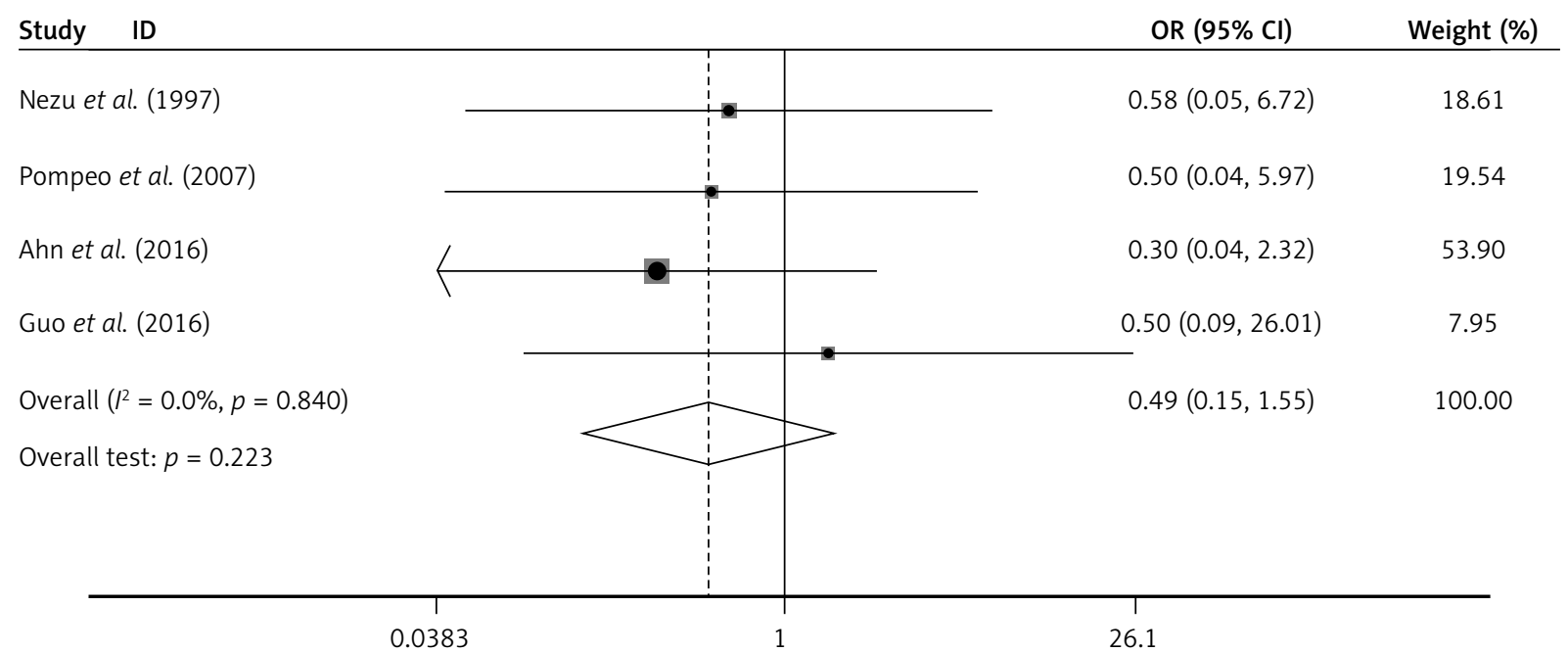

Figure 7. The forest plot of OR and its $95 \% \mathrm{Cl}$ of recurrence between ELA group and GA group

hypercapnia, unexpected thoracic pain requiring additional local anesthesia, epidural hematoma, spinal cord injury and phrenic nerve palsy caused by inadvertently high anesthetic level [19, 21, 29]. What is worse, the process of ELA to GA conversion may induce significant mediastinal or lung movement, persistent hypoxaemia, unstable hemodynamic status or uncontrolled bleeding [30]. Gonzalez-Rivas et al. stated that the rate of conversion to general anesthesia is less than 5\%, and strong adhesions, suboptimal analgesia, bleeding, intractable cough and so forth are the most common reasons for conversion [31]. However, these adverse effects of ELA are rare, and some measures can be adopted to deal with relevant complications. For instance, Chen et al. reported that intrathoracic vagal blockade was produced by infiltration of $2 \mathrm{ml}$ of $0.25 \%$ bupivacaine and it could inhibit the cough reflex for $3 \mathrm{~h}$ or even longer [10].

Since there is still controversy about the role of ELA in VATS for pneumothorax, we conducted this meta-analysis to comprehensively assess and compare the results of the ELA group and GA group based on the related studies.

After analyzing the relevant results, we reached two main conclusions. On the one hand, there is no extreme difficulty or complex operation in the surgical procedures of the ELA group because of the lack of significant difference in operative time between the ELA group and GA group. An experienced surgi- 
cal team is the key to success. The VATS operation under ELA by practiced surgeons and skilled anesthesiologists could be done with a low incidence of conversion to general anesthesia and complications $[31,32]$. On the other hand, the patients in the ELA group, compared with GA, were not provided additional security and could uneventfully leave the hospital in several days. Hence the feasibility and safety of thoracoscopic surgery of spontaneous pneumothorax under ELA is demonstrated by these two conclusions.

Additionally, we had some other findings. The ELA group had a shorter global operating room time because it saved not only the preoperative time, but also the postoperative time. Wu et al. suggested that the ELA group had a shorter anesthesia induction time because it did not need time on tracheal intubation and subsequent bronchoscopic examination [12]. Also, the ELA group without muscle relaxants could expedite postoperative recovery $[30,33]$. Secondly, Ahn et al. recorded 2 hospital deaths in the ELA group, of which one had destructive lung due to multiple huge bullae and resulted in mediastinal shift, and another one had underlying unusual interstitial pneumonia [21]. Thus it remains controversial whether patients with severe underlying disease could undergo ELA. According to some reported studies, American Society of Anesthesiologists (ASA) scores of greater than 3, bleeding disorders, sleep apnea, obesity (body mass index > $30 \mathrm{~kg} / \mathrm{m}^{2}$ ), extensive pleural adhesions, presence of epidural puncture contraindication, expected difficult airway management (such as airway hyper-reactivity) and tumors $>6 \mathrm{~cm}$ were considered as contraindications of thoracoscopic surgery under ELA [31, 34, 35].

\section{Limitations}

Firstly, publication bias was tested by Begg's test and Egger's test and there was no significant bias (Begg's test: $p=0.462$; Egger's test: $p=0.374$, Figure 8). However, the result of publication bias was not significantly meaningful, because of lack of enough studies available for this analysis. Therefore publication bias was unavoidable. Secondly, both randomized controlled trials and observational studies were included in this analysis at the same time, which could affect the validity of the results. The third limitation was the small sample size of most relevant studies. What is worse, het-

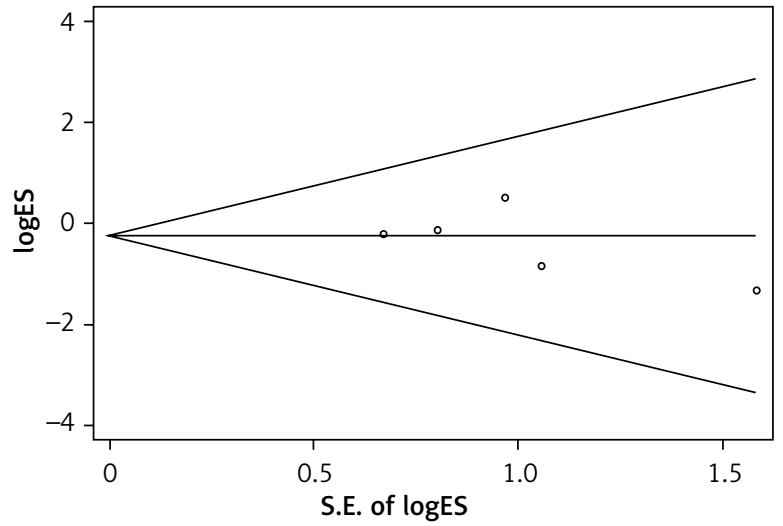

Figure 8. The funnel plot of the included studies for overall analysis of complications between ELA group and GA group (Begg's test: $p=0.462$; Egger's test: $p=0.374$ )

erogeneities were seen in the analysis of operative time and hospital stay. The origin of heterogeneities may be that these data come from different studies - different districts, different times, and different surgical teams.

\section{Conclusions}

ELA and GA had similar operative time, hospital stay, complications, air leak, recurrence and perioperative mortality. However, ELA had a shorter global operating room time and lower incidence of related complications of GA. This analysis indicates that the thoracoscopic surgery of spontaneous pneumothorax under ELA is feasible and safe. In view of eliminating the correlated limitations and even renewing our conclusion, a well-designed and multi-centre randomized controlled trial in a large patient population with a long-term follow-up period is strongly and urgently needed.

\section{Conflict of interest}

The authors declare no conflict of interest.

\section{References}

1. MacDuff A, Arnold A, Harvey J; BTS Pleural Disease Guideline Group. Management of spontaneous pneumothorax: British Thoracic Society pleural disease guideline 2010. Thorax 2010; 65 (Suppl 2): ii18-31.

2. Whitehead T, Slutsky AS. The pulmonary physician in critical care * 7 : ventilator induced lung injury. Thorax 2002; 57: 635-42.

3. Pompeo E, Mineo D, Rogliani P, et al. Feasibility and results of awake thoracoscopic resection of solitary pulmonary nodules. Ann Thorac Surg 2004; 78: 1761-8. 
4. Mineo TC. Epidural anesthesia in awake thoracic surgery. Eur J Cardiothorac Surg 2007; 32: 13-9.

5. Licker M, Fauconnet P, Villiger Y, et al. Acute lung injury and outcomes after thoracic surgery. Curr Opin Anaesthesiol 2009; 22: 61-7.

6. Pinheiro de Oliveira R, Hetzel MP, dos Anjos Silva M, et al. Mechanical ventilation with high tidal volume induces inflammation in patients without lung disease. Crit Care 2010; 14: R39.

7. Herndon B, Yagan M, Reisz G, et al. Metabolic and biochemical responses of the healthy human lung to nonthoracic surgery. Lung 2008; 186: 63-70.

8. Nezu K, Kushibe K, Tojo T, et al. Thoracoscopic wedge resection of blebs under local anesthesia with sedation for treatment of a spontaneous pneumothorax. Chest 1997; 111: 230-5.

9. Lesser TG. Laser application enables awake thoracoscopic resection of pulmonary nodules with minimal access. Surg Endosc 2012; 26: 1181-6.

10. Chen JS, Cheng YJ, Hung MH, et al. Nonintubated thoracoscopic lobectomy for lung cancer. Ann Surg 2011; 254: 1038-43.

11. Pompeo E, Mineo TC. Awake pulmonary metastasectomy. J Thorac Cardiovasc Surg 2007; 133: 960-6.

12. Wu CY, Chen JS, Lin YS, et al. Feasibility and safety of nonintubated thoracoscopic lobectomy for geriatric lung cancer patients. Ann Thorac Surg 2013; 95: 405-11.

13. Liu J, Cui F, Pompeo E, et al. The impact of non-intubated versus intubated anaesthesia on early outcomes of video-assisted thoracoscopic anatomical resection in non-small-cell lung cancer: a propensity score matching analysis. Eur I Cardiothorac Surg 2016; 50: 920-5.

14. Mineo TC, Sellitri F, Tacconi F, et al. Quality of life and outcomes after nonintubated versus intubated video-thoracoscopic pleurodesis for malignant pleural effusion: comparison by a casematched study. J Palliat Med 2014; 17: 761-8.

15. Pompeo E, Rogliani P, Tacconi F, et al. Awake Thoracic Surgery Research Group. Randomized comparison of awake nonresectional versus nonawake resectional lung volume reduction surgery. J Thorac Cardiovasc Surg 2012; 143: 47-54.

16. Liu J, Li S, Shen J, et al. Non-intubated resection and reconstruction of trachea for the treatment of a mass in the upper trachea. J Thorac Dis 2016; 8: 594-9.

17. Li S, Liu J, He J, et al. Video-assisted transthoracic surgery resection of a tracheal mass and reconstruction of trachea under non-intubated anesthesia with spontaneous breathing. I Thorac Dis 2016; 8: 575-85.

18. Pompeo E, Tacconi F, Mineo TC. Awake video-assisted thoracoscopic biopsy in complex anterior mediastinal masses. Thorac Surg Clin 2010; 20: 225-33.

19. Pompeo E, Tacconi F, Mineo D, et al. The role of awake video-assisted thoracoscopic surgery in spontaneous pneumothorax. J Thorac Cardiovasc Surg 2007; 133: 786-90.

20. Noda M, Okada Y, Maeda S, et al. Is there a benefit of awake thoracoscopic surgery in patients with secondary spontaneous pneumothorax? J Thorac Cardiovasc Surg 2012; 143: 613-6.

21. Ahn HY, Kim YD, Cho JS, et al. Thoracoscopic surgery under epidural anesthesia for intractable secondary spontaneous pneumothorax. Asian J Surg 2017; 40: 285-9.

22. Guo Z, Yin W, Zhang X, et al. Primary spontaneous pneumothorax: simultaneous treatment by bilateral non-intubated videothoracoscopy. Interact Cardiovasc Thorac Surg 2016; 23: 196-201.

23. Jin W, Li YP. The selection of a summary statistic for use in meta-analysis. Chin J Evid Based Med 2007; 7: 606-13.

24. Egger M, Davey Smith G, Schneider M, et al. Bias in meta-analysis detected by a simple, graphical test. BMJ 1997; 315: 629-34.

25. Mukaida T, Andou A, Date H, et al. Thoracoscopic operation for secondary pneumothorax under local and epidural anesthesia in high-risk patients. Ann Thorac Surg 1998; 65: 924-6.

26. Iwata Y, Hamai Y, Koyama T. Anesthetic management of nonintubated video-assisted thoracoscopic surgery using epidural anesthesia and dexmedetomidine in three patients with severe respiratory dysfunction. J Anesth 2016; 30: 324-7.

27. Hung WT, Hsu HH, Hung MH, et al. Nonintubated uniportal thoracoscopic surgery for resection of lung lesions. J Thorac Dis 2016; 8 (Suppl 3): S242-50.

28. Irons JF, Miles LF, Joshi KR, et al. Intubated versus nonintubated general anesthesia or video-assisted thoracoscopic surgery a case control study. J Cardiothorac Vasc Anesth 2017; 31: 411-7.

29. Pompeo E, Mineo TC. Awake operative videothoracoscopic pulmonary resections. Thorac Surg Clin 2008; 18: 311-20.

30. Pompeo E. Awake thoracic surgery - is it worth the trouble? Semin Thorac Cardiovasc Surg 2012; 24: 106-14.

31. Gonzalez-Rivas D, Aymerich H, Bonome C, et al. From open operations to nonintubated uniportal video-assisted thoracoscopic lobectomy: minimizing the trauma to the patient. Ann Thorac Surg 2015; 100: 2003-5.

32. Gonzalez-Rivas D, Bonome C, Fieira E, et al. Non-intubated video-assisted thoracoscopic lung resections: the future of thoracic surgery? Eur J Cardiothorac Surg 2016; 49: 721-31.

33. Liu J, Cui F, Li S, et al. Non-intubated videoassisted thoracoscopic surgery under epidural anesthesia compared with conventional anesthetic option: a randomized control study. Surg Innov 2015; 22: 123-30.

34. Chen KC, Cheng YJ, Hung MH, et al. Nonintubated thoracoscopic lung resection: a 3-year experience with 285 cases in a single institution. J Thorac Dis 2012; 4: 347-51.

35. Dong Q, Liang L, Li Y, et al. Anesthesia with nontracheal intubation in thoracic surgery. J Thorac Dis 2012; 4: 126-30.

Received: 24.04.2017, accepted: 8.06.2017. 